\title{
Safety and Efficacy of Isotonic (0.9\%) vs. Hypotonic (0.18\%) Saline as Maintenance Intravenous Fluids in Children: A Randomized Controlled Trial
}

\author{
Ahmar Shamim, Kamran Afzal and S Manazir Ali \\ From Department of Pediatrics, Jawaharlal Nehru Medical College, Aligarh Muslim University, Aligarh, Uttar Pradesh, India. \\ Correspondence to: Dr Ahmar Shamim, Assistant Professor, Mahatma Gandhi Mission Medical College, Navi Mumbai, \\ Maharashtra,India.ahmar_shamim@yahoo.com \\ Received: July 18, 2014; Initial review: September 26, 2014; Accepted: October 09, 2014.
}

Objective: To compare the safety and efficacy of isotonic versus hypotonic maintenance fluid in children.

Design: Randomized controlled trial.

Setting: Tertiary-level teaching hospital.

Participants: 60 children (age 0.5 to 12 years) who were admitted and anticipated to receive intravenous fluid for the next 48 hours.

Intervention: Hypotonic fluid (Standard maintenance volume as $0.18 \% \mathrm{NaCl}$ in $5 \%$ dextrose) or Isotonic fluid $(60 \%$ Standard maintenance volume as $0.9 \% \mathrm{NaCl}$ solution in $5 \%$ dextrose).

Outcome measures: Primary: Incidence of hyponatremia. Secondary: Serum sodium, serum osmolality, blood sugar, blood urea, serum creatinine, serum potassium, serum chloride, $\mathrm{pH}$, urine output, change in weight, morbidity and death. Results: At 24 hours, hyponatremia was noted in $7(24 \%)$ patients in the ecommendation for the use of a hypotonic saline solution ( $0.18 \%$ saline in $5 \%$ dextrose) in children is still a debated subject despite half a century of its practice [1]. Reports of symptomatic hyponatremia in hospitalized surgical and non-surgical pediatric patients - caused primarily by various non-osmotic release of vasopressin, but contributed by electrolyte-free water input in a proportion of cases - have fueled these debates [2-5]. Use of conventional volume maintenance isotonic saline has been shown to reduce the incidence of hyponatraemia [6]. Using indirect calorimetric measurements, energy expenditure in critically ill children may be as low as 50$60 \mathrm{kcal} / \mathrm{kg} /$ day [7]. Consequently, fluid requirement, which is directly proportional to the actual energy expenditure, is much less than previously assumed in critically ill children for a variety of reasons such as physical immobility, the use of muscle relaxants and sedatives, mechanical ventilation, and additional factors such as nonessential or facultative metabolism [8]. Therefore, we hypothesized that use of reduced volume isotonic maintenance fluid would decrease the incidence isotonic and $16(55 \%)$ in hypotonic group $(P=0.031)$. At 48 hours, hyponatremia was noted in $4(14 \%)$ and $13(45 \%)$ patients in isotonic and hypotonic group, respectively $(P=0.02)$. There was significant change in sodium levels in both isotonic $(P=0.036)$ and hypotonic $(P<0.001)$ intervention groups. The peak fall in mean serum sodium level was noted at 24 hours $(-6.5,95 \% \mathrm{Cl}$ : $-3.5,-9.6$ $\mathrm{mEq} / \mathrm{L} ; P<0.001)$ in hypotonic group. In isotonic group, there was significant increase between 24 and 48 hours $(4.3,95 \% \mathrm{Cl}$ : 0.1 , $8.4 \mathrm{mEq} / \mathrm{L} ; P=0.04$ ).

Conclusions: Reduced volume isotonic fluid results in fewer episodes of hyponatremia than hypotonic fluid in sick children during the first 48 hours of intravenous fluid therapy.

Keywords: Hyponatremia, Intravenous fluids, Normal saline, Parenteral fluid therapy.

Trial registration: CTRI/2010/091/000398. of hyponatremia in sick children, when compared to hypotonic fluid. We compared the efficacy and safety of isotonic fluid $(0.9 \% \mathrm{NaCl}$ in $5 \%$ dextrose) at the rate of $60 \%$ of daily fluid requirement versus hypotonic fluid $(0.18 \% \mathrm{NaCl}$ in $5 \%$ dextrose $)$ at the rate of standard maintenance volume in sick children.

Accompanying Editorials: Pages 963-66.

\section{Methods}

This open-label randomized controlled trial was conducted in the Pediatric ward of Jawahar Lal Nehru Medical College Hospital in Aligarh, India from November 2009 to October 2010. Children in the age group of 0.5 to 12 years, who were admitted and anticipated to receive intravenous fluid for the next 48 hours, were considered for recruitment into the study. Written informed consent was obtained from the parent or guardian of all patients before enrolment. The study protocol was approved by the Institute Ethics Committee.

Children with hyponatremia (serum sodium $<130$ 
$\mathrm{mEq} / \mathrm{L}$ ), hypernatremia (serum sodium >150 mEq/L), acute gastroenteritis, hemodynamic instability (shock, myocarditisbcongestive heart failure), acute or chronic kidney disease, a history/evidence of cardiac dys-function, uncontrolled seizures, severe developmental delay, diabetes mellitus or insipidus, and severe malnutrition, were excluded. Children with pre-existing hypertension, diuretic use, edema, or known adrenal dysfunction, or those who had received intravenous fluid within preceding 3 hours were also excluded.

All eligible patients were randomized to receive either hypotonic saline solution ( $0.18 \% \mathrm{NaCl}$ in $5 \%$ dextrose), at the rate of standard maintenance volume or isotonic saline solution $(0.9 \% \mathrm{NaCl}$ solution in $5 \%$ dextrose), at the rate of $60 \%$ of standard maintenance volume. Maintenance fluid volume for administration was calculated using Holliday and Segar formula [1]. Both groups also received $1 \mathrm{~mL}$ of potassium chloride per $100 \mathrm{~mL}$ of intravenous fluids. Randomization sequence was generated (block randomization, size 4) and maintained by a colleague not directly involved in this trial using an online computer program. The allocated intervention was kept in sequentially labeled sealed opaque envelopes to be opened at the time of randomization.

Baseline demographic, anthropometric and laboratory characteristics were noted at enrolment. All patients were monitored clinically for symptoms and signs of dysnatremia, and signs of fluid overload or dehydration throughout the study period. Clinical assessment, including weight and urine output was done every 12 hours. Laboratory measurements included 12-hourly serum sodium, serum potassium, serum chloride, $\mathrm{pH}$ (Combiline Eschweiler blood gas and electrolyte analyzer), blood urea, serum creatinine and blood sugar. Serum osmolality (by freeze point depression using Osmomat-30, Gonotech, Germany) was done at 0, 24 and 48 hours. Participants who developed symptomatic dysnatremia (serum sodium $<130$ or $>150 \mathrm{mEq} / \mathrm{L}$ ) or asymptomatic dysnatremia (serum sodium $<125$ or $>155 \mathrm{mEq} / \mathrm{L}$ ), dehydration/features of hypervolemia, require fluid boluses for volume resuscitation, weight loss of $>5 \%$, increase in blood urea/serum creatinine $>10 \%$ from baseline or urine output $<1 \mathrm{~mL} / \mathrm{kg} / \mathrm{hr}$ at any stage were excluded from the study.

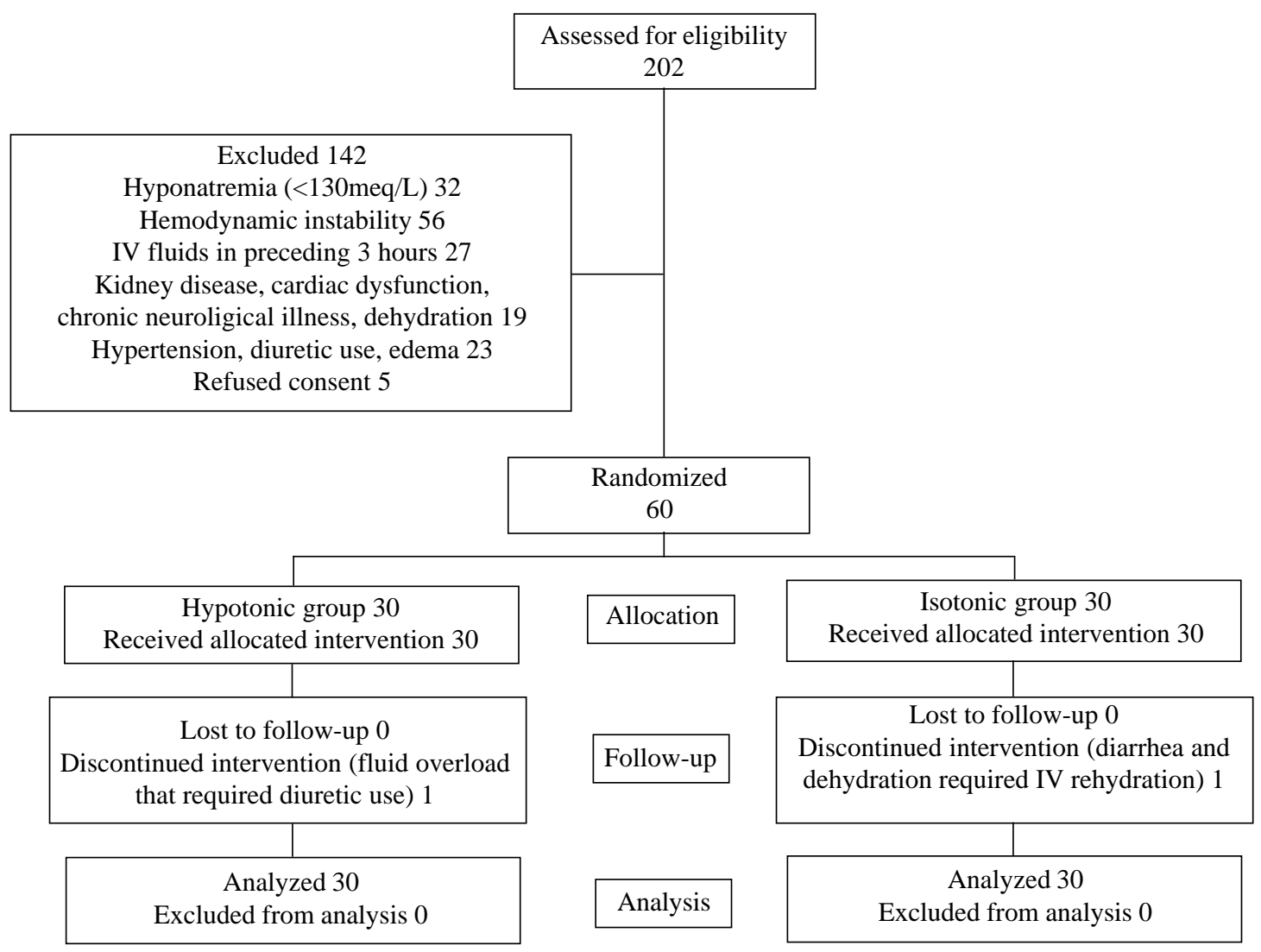

FIG. 1 Flow of patients in the study. 
The primary outcome of the study was the incidence of hyponatremia (defined as serum sodium $<130 \mathrm{mEq} / \mathrm{L}$ ). Incidence of hypernatremia, serum sodium, serum osmolality, blood sugar, blood urea, serum creatinine, serum potassium, serum chloride, $\mathrm{pH}$, urine output, change in weight, morbidity (seizures, cerebral edema or other neurological manifestations attributable to dysnatremias) and death were secondary outcome measures.

As the previously reported incidence of hyponatremia is quite variable, we calculated the sample size from our pilot data of 10 patients per intervention group; hyponatremia was observed in 5 patients on hypotonic fluid (HF) and 1 in isotonic fluid (IF). A sample size of 25 patients per group was calculated to be adequate to reject the null hypothesis with a power of $90 \%$ and an alpha error of 0.05 . To account for a $15 \%$ loss to follow-up, we decided to enroll 30 patients per intervention group.

The data were analyzed with SPSS version 17.0 software. Analysis was done on an intention-to-treat basis. Ordinal data was compared using Fisher's exact test or Chi-square test. Independent sample t-test was used to compare difference in serum sodium, serum osmolality, urinary outputs, percentage change in weight, and other secondary variables, of the two groups in the study. Significance was taken at $\mathrm{P}$ value of $<0.05$.

\section{Results}

During the study period, a total of 480 consecutive patients were assessed of which 202 met the eligibility criteria. Of these, 142 children had to be excluded (Fig. 1). Thus a total of 60 children were randomized, 30 to each treatment intervention group. Mean (SD) age of the study population was 53.8 (35.5) months; 33 (55\%) were male. Baseline characteristics did not differ significantly between the two intervention groups (Table I). Of the 15 cases with infections, diagnosis was enteric fever $(n=4)$, complicated malaria $(n=8)$ or sepsis $(n=3)$. The study was discontinued within 24 hours in 2 patients (one from each group); one patient in IF developed acute gastroenteritis with dehydration requiring intravenous rehydration and the other patient in HF developed clinical features of fluid overload requiring diuretics.

Hyponatremia: The incidence of hyponatremia was $33.3 \%(\mathrm{n}=10)$ in the IF group and $70 \%(\mathrm{n}=21)$ in the HF group (RR 0.48, (95\%CI, 0.27, 0.83; $P=0.01)$. The number of children with hyponatremia at 12, 24, 36 and 48 hours were 3, 7, 3 and 4 in IF and 4, 16, 14 and 13 in HF. The incidence was significantly different at 24 ( $P=0.031), 36(P=0.003)$ and 48 hours $(P=0.022)$.

Of the patients who developed hyponatremia, 9 had values below $125 \mathrm{mEq} / \mathrm{L}$ ( 2 in IF and 7 in HF). The

\begin{tabular}{lll} 
TABLE I & $\begin{array}{c}\text { BASELINE } \\
\text { CHARACTERISTICS OF PARTICIPANTS }\end{array}$ & BIOCHEMICAL \\
\hline Parameter & $\begin{array}{c}\text { Isotonic } \\
\text { group } \\
(N=30)\end{array}$ & $\begin{array}{c}\text { Hypotonic } \\
\text { group } \\
(N=30)\end{array}$ \\
\hline Age (mo) & $53.1(39.5)$ & $54.4(31.7)$ \\
Sex (M:F) & $17: 13$ & $16: 14$ \\
Weight-for-age, \% & $79.5(9.0)$ & $77.7(6.7)$ \\
Body mass index, Kg/m² & $14.6(1.2)$ & $13.9(1.0)$ \\
Body surface area, m ${ }^{2}$ & $0.6(0.20)$ & $0.6(0.16)$ \\
Serum sodium, mEq/L & $135.7(4.2)$ & $136.3(3.5)$ \\
Serum osmolality, mOSm/L & $291.4(10.0)$ & $293.8(8.5)$ \\
Blood sugar, mg/dL & $104.3(13.9)$ & $99.3(12.7)$ \\
Blood urea, mg/dL & $39.6(8.6)$ & $44.2(9.5)$ \\
Serum creatinine, mg/dL & $0.70(0.08)$ & $0.77(0.09)$ \\
Serum potassium, mEq/L & $3.9(0.8)$ & $4.1(0.6)$ \\
Serum chloride, mEq/L & $99.8(6.3)$ & $101.3(6.6)$ \\
pH & $7.36(0.06)$ & $7.36(0.06)$ \\
Diagnosis at admission & & \\
Meningo-encephalitis & 9 & 11 \\
Acute respiratory illness & 12 & 11 \\
Infections & 8 & 7 \\
Acute hepatitis & 1 & 1 \\
\hline
\end{tabular}

All values in mean (SD); $P>0.05$ for all parameters

numbers that had serum sodium level $<125 \mathrm{mEq} / \mathrm{L}$ were 3 (2 in HF) at 24 hours and 5 (4 in HF) at 48 hours. There was no patient with serum sodium level below $120 \mathrm{mEq} /$ L. Using serum sodium levels below $135 \mathrm{mEq} / \mathrm{L}$ to define hyponatremia, 25 in $\mathrm{HF}$ and 16 in IF were hyponatremic at 24 hours $(P=0.009)$ whereas at 48 hours, 20 in HF and 6 in IF were hyponatremic $(\mathrm{P}<0.001)$. Hypernatremia was noted at 48 hours in $3 \mathrm{IF}(P=0.27)$.

Serum sodium: Significant changes in sodium levels were observed in both intervention groups (Table II). In the IF group, mean serum sodium was constant over initial 24 hours; thereafter, an increase was observed between 24 and 48 hours $(4.3 \mathrm{meq} / \mathrm{L}, 95 \% \mathrm{CI}: 0.1,8.4 \mathrm{meq} / \mathrm{L}$; $(P=0.04)$. In HF group, there was a significant decline in mean serum sodium level at 24 hours $(6.5 \mathrm{meq} / \mathrm{L}, 95 \%$ CI: 3.5, $9.6 \mathrm{mEq} / \mathrm{L} ;(P<0.001)$, and the decline persisted till the end of study (4.9 meq/L, 95\%CI: 1.9, $7.9 \mathrm{mEq} / \mathrm{L}$; $(P<0.001)$.

Serum osmolality: In IF group, serum osmolality remained constant for the initial 24 hours and thereafter it increased (mean increase at 48 hours $9.3 \mathrm{mos} \mathrm{mol} / \mathrm{L}, 95 \%$ CI: $1.3,17.2 \mathrm{mos} \mathrm{mol} / \mathrm{L} ; P=0.02$ ). In HF group, there was a decline starting at 24 hours (14 mos mol/L, 95\% CI: 8.1, 20 mos moL; $P<0.001$ ) and persisted till the end of the 
observation period (11.7 mosmol/L, 95\% CI: 5.7, 17.6 mosmol/L; $P<0.001)$.

Blood urea/creatinine, serum potassium, blood sugar, chloride and $\mathrm{pH}$ : Changes in blood urea and creatinine were not significant, except for a significant $(P<0.001)$ decline in creatinine in HF group. There were no episodes of hypo - or hyper-kalemia. Changes in blood sugar, serum chloride and $\mathrm{pH}$ in either group were not significant.

Weight change: In IF group, there was progressive fall in weight, and peak fall from baseline weight (2.6\%, 95\% CI: $1.9 \%, 3.3 \%)$ was at 48 hours $(P<0.001)$. In HF group, there was persistent rise in weight and maximum rise (2.8\%, 95\% CI: $1.0,3.7 \%)$ was noted at 48 hours $(P<0.001)$.

Urine output: Change in urine output in IF group was not significant $(P=0.2)$ whereas in HF group, it increased (0.15 mL/Kg/h, 95\%CI: 0.06, 0.24 mL/kg/h; $P<0.001)$.
No episode of oligouria or anuria occurred in either group.

Symptomatic dysnatremia and mortality: There were no symptoms attributable to dysnatremia in either intervention group. No mortality occurred in any of the group during the observation period.

\section{Discussion}

The incidence of hyponatremia with the use of hypotonic intravenous fluids has ranged from $7.6 \%$ to $57 \%$ [9-13]. We observed unusually high incidence of $70 \%$ hyponatremia with the use of hypotonic 'standard volume' maintenance fluid in a selected group of nonsurgical pediatric patients. In this study we tested the efficacy of reduced volume isotonic maintenance fluid, and confirm significant reduction in the risk of hyponatremia. However, in the absence of a comparative IF group receiving unrestricted maintenance volume in this study, it is difficult to assess the contribution of fluid

TABLE II LABORATORY MEASUREMENTS In STUdy PARTICIPANTS*

\begin{tabular}{|c|c|c|c|c|}
\hline Laboratory investigations" & Time (hours) & $I F$ & $H F$ & Pvalue \\
\hline \multirow[t]{3}{*}{ Serum sodium (mEq/l) } & 0 & $135.7(134.1,137.4)$ & $136.3(135.0,137.7)$ & 0.62 \\
\hline & 24 & $134.6(132.5,136.8)$ & $129.7(128.3,131.1)$ & $<0.001$ \\
\hline & 48 & $138.9(136.0,141.8)$ & $131.4(129.2,133.5)$ & $<0.001$ \\
\hline \multirow[t]{3}{*}{ Serum osmolality(mOsm/l) } & 0 & $291.4(287.7,295.4)$ & $293.8(290.8,297.3)$ & 0.32 \\
\hline & 24 & $290.9(286.1,295.7)$ & $279.8(276.6,283.0)$ & $<0.001$ \\
\hline & 48 & $300.7(294.8,306.6)$ & $282.2(277.8,286.6)$ & $<0.001$ \\
\hline \multirow[t]{3}{*}{ Blood urea(mg/dL) } & 0 & $39.6(36.4,43.0)$ & $44.2(40.8,48.1)$ & 0.06 \\
\hline & 24 & $41.8(38.2,45.4)$ & $42.0(38.9,45.2)$ & 0.92 \\
\hline & 48 & $43.5(39.6,47.5)$ & $40.0(37.1,43.0)$ & 0.15 \\
\hline \multirow[t]{3}{*}{ Serum creatinine (mg/dL) } & 0 & $0.70(0.67,0.73)$ & $0.77(0.73,0.80)$ & 0.06 \\
\hline & 24 & $0.71(0.69,0.73)$ & $0.72(0.69,0.75)$ & 0.66 \\
\hline & 48 & $0.71(0.68,0.74)$ & $0.69(0.66,0.71)$ & 0.19 \\
\hline \multirow[t]{3}{*}{ Serum potassium(mEq/L) } & 0 & $3.95(3.64,4.26)$ & $4.12(3.91,4.34)$ & 0.34 \\
\hline & 24 & $4.18(3.89,4.47)$ & $3.87(3.63,4.11)$ & 0.09 \\
\hline & 48 & $4.45(4.09,4.80)$ & $3.63(3.27,3.99)$ & 0.01 \\
\hline \multirow[t]{3}{*}{ Serum chloride(mEq/L) } & 0 & $99.8(97.4,102.1)$ & $101.3(98.9,103.8)$ & 0.35 \\
\hline & 24 & $101.4(98.9,104.0)$ & $99.9(97.5,102.3)$ & 0.31 \\
\hline & 48 & $102.8(99.7,106.0)$ & $99.3(96.6,102.0)$ & 0.17 \\
\hline \multirow[t]{3}{*}{$\mathrm{pH}$} & 0 & $7.36(7.34,7.39)$ & $7.36(7.34,7.38)$ & 0.99 \\
\hline & 24 & $7.35(7.32,7.37)$ & $7.36(7.34,7.39)$ & 0.28 \\
\hline & 48 & $7.32(7.30,7.36)$ & $7.38(7.35,7.40)$ & 0.01 \\
\hline \multirow[t]{3}{*}{ Blood sugar(mg/dL) } & 0 & $104.3(99.7,110.1)$ & $99.3(94.2,103.9)$ & 0.17 \\
\hline & 24 & $108.8(104.9,112.8)$ & $99.6(96.4,102.8)$ & $<0.001$ \\
\hline & 48 & $110.3(107.3,113.4)$ & $101.5(98.1,104.9)$ & $<0.001$ \\
\hline
\end{tabular}

ฯ Mean (95\% confidence interval), IF =Isotonic group, $H F=$ Hypotonic group; *N=30 at $0 \mathrm{hr}$ and $N=29$ in both groups at 24 and 48 hr; \# Independent Sample t-test for IF versus HF. 


\section{What is AlReady KnOWn?}

- Standard volume maintenance isotonic fluids reduce the incidence of hyponatremia in comparison to hypotonic maintenance fluids in children.

\section{WhAt ThIS StUdy AdDS?}

- Reduced volume isotonic maintenance fluids results in fewer episodes of hyponatremia in comparison to hypotonic maintenance fluids in non-surgical pediatric patients.

- The risk of hyponatremia is highest at 24 hours of intravenous fluid administration, and requires close monitoring.

restriction versus higher fluid sodium, to the improved sodium levels in the IF group.

Isotonic maintenance fluids have consistently been shown to lower the incidence of hyponatremia [6,12-16]. Reducing the volume of administered fluid has also been shown to decrease the risk of hyponatremia in children with free-water excess [13]. Kannan, et al. [13] have previously shown that hyponatremia is less common with the use of isotonic saline in standard volume (1.72\%) as well as with reduced volume hypotonic saline solution (3.8\%) when compared to standard volume hypotonic fluid (14.3\%). Young and Keeley [14], however, reported that fluid type but not rate was significantly associated with hyponatremia in surgical pediatric patients.

Similar to findings from our study, Saba, et al. [16] also did not find a change in serum sodium in the first 12 hours in both intervention groups (isotonic or $0.45 \% \mathrm{NaCl}$ ). Thus maximum risk of hyponatremia with use of either fluid is at 24 hours, and warrants close clinical and laboratory monitoring. Similar to our observations, Neville, et al. [12] observed that serum osmolality remained constant in patients receiving isotonic fluids $(0.9 \% \mathrm{NS})$, whereas there was significant fall after 4 hours of fluid therapy in those receiving $0.45 \%$ saline. Thus, continued administration of restricted volume isotonic fluids beyond 24 hours may impose risk of dehydration and hypernatremia.

Our study had certain limitations. First, the study excluded all surgical patients as well as serious medical conditions because of variability in their fluid requirements. In usual clinical settings, these patients commonly require parenteral maintenance fluid administration and are more prone to develop dysnatremia. Second, a comparative IF arm, receiving full maintenance fluid would have been useful to ascertain the influence of fluid restriction over and above isotonic saline administration. Third, we did not measure urinary electrolytes/osmolality and serum anti-diuretic hormone levels. These would have been important for assessing volume status and free water clearance. Lastly, since none of the patients in either group received other fluids, it is difficult to extrapolate the results of this trial to usual clinical scenario wherein patients often require deficit replacements, partial maintenance fluid and sodium from other sources. Further studies with a larger sample size and an additional control arm using standard volume isotonic fluids may determine the overall benefit and safety of volume reduction.

To conclude, intravenous isotonic fluid at the rate of $60 \%$ of daily maintenance fluid requirement results in fewer episodes of hyponatremia than standard volume maintenance hypotonic fluid during 48 hours of treatment without significant increase in the incidence of hypernatremia. Continued administration of isotonic fluids beyond 24 hours, deserve close monitoring and modification of fluid therapy.

Contributors: AS: concept of study, data collection, analysis, drafting and revision of manuscript; KA: concept, design and supervision of the work; analysis of data; and drafting and revision of manuscript for important intellectual content; SMA: supervision of the work, data analysis and revision of manuscript. KA will act as the guarantor.

Funding: None; Competing Interests: None stated.

\section{REFERENCES}

1. Holliday MA, Segar ME. Maintenance need for water in parenteral fluid therapy. Pediatrics. 1957;19:823-32.

2. Anderson RJ. Hospital associated hyponatremia. Kidney lnt. 1986;29:1237-47.

3. Arieff AI, Ayus JC, Fraser CL, Hyponatraemia and death or permanent brain damage in healthy children. BMJ. 1992;304:1218-22.

4. Halberthal M, Halperin ML, Bohn D. Acute hyponatremia in children admitted in hospital. BMJ. 2001;322:780-2.

5. Holliday MA, Friedman A, Segar ME, Chesney R, Finberg L. Acute hospital induced hyponatremia in children: A physiological approach. J Pediatr. 2004;145:584-7.

6. Montañana PA, Modesto i Alapont V, Ocón AP, López PO, López Prats JL, et al. The use of isotonic fluid as maintenance therapy prevents iatrogenic hyponatremia in pediatrics: A randomized, controlled open study. Pediatr Crit Care Med. 2008;9:658-9.

7. Briassoulis G, Venkataraman S, Thompson AE. Energy expenditure in critically ill children. Pediatr Crit Care Med. 
2000;28:1166-72.

8. Choong K, Bohn D. Maintenance parenteral fluids in the critically ill child. J Pediatr (Rio J). 2007; 83:S3-10.

9. Duke T, Mokela D, Frank D, Michael A, Paulo T, Mgone $\mathrm{J}$, et al. Management of meningitis in children with oral fluid restriction or intravenous fluid at maintenance volumes: a randomized trial. Ann Trop Pediatr. 2002;22:145-57.

10. Hoorn EJ, Geary D, Robb M, Halperin ML, Bohn D. Acute hyponatremia related to intravenous fluid administration in hospitalized children: An observational study. Pediatrics. 2004;113:1279-84.

11. Brazel P, McPhee IP. Inappropriate secretion of antidiuretic hormone in postoperative scoliosis patients: The role of fluid management. Spine. 1996;21:727.

12. Neville K, Verge C, Rosenberg A, O’Meara M, Walker J. Isotonic is better than hypotonic saline for intravenous rehydration of children with gastroenteritis: A prospective randomized study. Arch Dis Child. 2006;91:226-32.

13. Kannan L, Lodha R, Vivekanandhan S, Bagga A, Kabra SK, Kabra $M$. Intravenous fluid regimen and hyponatraemia among children: A randomized controlled trial. Pediatr Nephrol. 2010;25:2303-9.

14. Yung M, Keeley S. Randomised controlled trial of intravenous maintenance fluids. J Paediatr Child Health. 2009;45:9-14.

15. Choong K, Arora S, Cheng J, Farrokhyar F, Reddy D, Thabane L, et al. Hypotonic versus isotonic maintenance fluids after surgery in children: A randomized controlled trial. Pediatrics. 2011;128:857-66.

16. Saba TG, Fairbairn J, Houghton F, Laforte D, Foster BJ. A randomized controlled trial of isotonic versus hypotonic maintenance intravenous fluids in hospitalized children. BMC Pediatr. 2011;11:82.

AdVERTISEMENT

\section{Opt - out of Hard Copies of Indian Pediatrics Join us to save paper, and reduce carbon footprint and cost}

Indian Pediatrics is freely available online - a rarity for such an indexed journal (indexed with Science citation index) in the world. The new issue is uploaded in the first week of every month at our website www.indianpediatrics.net. The 'Table of Contents' alert, with links to full text articles, is now being mailed to all IAP members. The articles are easily readable in PDF format on mobile and tablet device. Soon, we will also be launching an 'App' of Indian Pediatrics for more reader-friendly access to our online edition. We have received several mails from senior members to discontinue with the practice of sending print version of the journal. However, we are continuing with it as a social service to the academicians, and on popular demand at the General Body Meetings of IAP. Printing and posting 22,000 copies every month involves huge expense and labour, besides not being environment friendly. With this background, we request IAP members to intimate us (by email to jiap@nic.in) whether they want to continue to receive the hard copy of the journal or if they want to opt out of hard copies. Those willing to opt out will also have the option of the electronic copy of the journal mailed to their account regularly. Members may mail their option in the following format:

\begin{tabular}{|l|l|l|l|l|}
\hline Name & $\begin{array}{l}\text { IAP } \\
\text { Membership } \\
\text { No. }\end{array}$ & $\begin{array}{l}\text { Do you want to } \\
\text { continue receiving } \\
\text { hard copy } \\
\text { (Yes/No) }\end{array}$ & $\begin{array}{l}\text { If you opt out of } \\
\text { hard copy, do you } \\
\text { want an electronic copy? } \\
\text { (Yes/No) }\end{array}$ & Remarks \\
\hline
\end{tabular}

